\title{
Silencing subtelomeric VSGs by Trypanosoma brucei RAP1 at the insect stage involves chromatin structure changes
}

\author{
Unnati M Pandya ${ }^{*}$, Ranjodh Sandhu, Bibo Li \\ From Epigenetics and Chromatin: Interactions and processes \\ Boston, MA, USA. 11-13 March 2013
}

\section{Background}

Trypanosoma brucei causes human African trypanosomiasis and undergoes antigenic variation to evade its mammalian host immune attack. Throughout its life cycle, T. brucei is covered with glycoproteins on its cell surface. When infecting a mammalian host, bloodstream form (BF) T. brucei expresses bloodstream expression site (BES)-linked variant surface glycoproteins (VSGs) in a monoallelic fashion to ensure effectiveness of antigenic variation. In the midgut of its insect vector, tsetse, procyclic form (PF) T. brucei expresses procyclins. After migration to the tsetse's salivary glands, T. brucei expresses metacyclic VSGs (mVSGs) to prepare for mammalian host infection. Regulation of VSG expression/silencing is critical for T. brucei virulence and its development. However the underlying mechanism of VSG regulation is not fully understood. Our lab identified a telomeric protein TbRAP1 as a key regulator of BES-VSGs in BF [1]. In this study we have further elucidated role of TbRAP1 in VSG regulation at PF stage and its possible underlying mechanism through chromatin remodeling.

\section{Materials and methods}

We depleted TbRAP1 in PF cells using an inducible RNA interference (RNAi) approach. Quantitative RT-PCR was performed to analyze changes in steady state mRNA levels of BES-lined and metacyclic VSGs after depletion of TbRAP1. In order to test the effect of TbRAP1 depletion on chromatin structure, Formaldehyde-Assisted Isolation of Regulatory Elements (FAIRE) analysis and Micrococcalnuclease digestion assays were performed.

\section{Results}

In this study, we found that depletion of TbRAP1 by RNAi led to derepression of $m V S G s$ at both the BF and PF stages. Similar to that observed in BF cells, silencing of BES-linked VSGs in PF cells also depends on TbRAP1. In addition, we found that silencing of BES-linked VSGs by TbRAP1 is stronger in PF cells than that in BF cells. Furthermore, removal of TbRAP1 led to a loosened chromatin structure at multiple loci in PF cells, particularly at the BES loci, but not in BF cells, indicating that different silencing mechanisms are used at different stages and that TbRAP1-mediated silencing may involve modulation of chromatin structure in PF cells.

\section{Conclusions}

Our observations confirm that TbRAP1 is a key VSG silencer and that TbRAP1 helps to determine the chromatin structure in multiple loci throughout the genome, particularly at BESs, at the insect stage.

\section{Acknowledgements \\ I thank Cleveland State University Research Council's Doctoral Dissertation Research Expense Award and Fellowship (DDREAFP) program for providing funds to attend this conference.}

Published: 18 March 2013

Reference

1. Yang X, Fiqueiredo LM, Espinal A, Okubo E, Li B: RAP1 is essential for silencing telomeric variant surface glycoprotein genes in Trypanosoma brucei. Cell 2009, 137:99-109.

doi:10.1186/1756-8935-6-S1-P63

Cite this article as: Pandya et al.: Silencing subtelomeric VSGs by

Trypanosoma brucei RAP1 at the insect stage involves chromatin structure changes. Epigenetics \& Chromatin 2013 6(Suppl 1):P63. 